\title{
Effetti di una dose di idrocortisone aggiuntiva sui parametri di performance in risposta a un esercizio fisico di breve durata in donne con iposurrenalismo primitivo
}

\author{
Emanuela Arvat ${ }^{1}$
}

Pubblicato online: 23 febbraio 2016

(C) Springer International Publishing AG 2016

Commento a:

Effect of a pre-exercise hydrocortisone dose on short-term physical performance in female patients with primary adrenal failure.

K. Simunkova, N. Jovanovic, E. Rostrup, P. Methlie, M. Øksnes, R. Miodini Nilsen, H. Hennø, M. Tilseth, K. Godang, A. Kovac, K. Løvas, E.S. Husebye. Eur J Endocrinol (2016) 174:97-105

Molti pazienti affetti da insufficienza surrenalica, sia primitiva che secondaria, avvertono la necessità di una somministrazione aggiuntiva di glucocorticoidi prima di eseguire un'attività fisico-sportiva. Tuttavia, i benefici clinici di tale pratica non sono mai stati dimostrati in studi controllati.

Lo scopo del presente studio è stato quello di valutare, in un gruppo di donne adulte affette da iposurrenalismo primitivo, gli effetti di una dose aggiuntiva di idrocortisone (10 mg per os) o di placebo un'ora prima di eseguire una sessione di attività sportiva di breve durata (test massimale al cicloergometro), confrontando i risultati con quelli ottenuti in un gruppo di controllo. Lo studio è stato eseguito in modo randomizzato e in doppio-cieco. Ai tempi 0,15 e 30 minuti dopo l'inizio della sessione sono stati valutati diversi parametri cardio-respiratori ed endocrino-metabolici in risposta all'esercizio.

La somministrazione di una dose aggiuntiva di idrocortisone prima della sessione non ha modificato, nelle donne con iposurrenalismo, la risposta all'esercizio fisico, in termini di modificazioni cardio-respiratorie o endocrinometaboliche. In particolare, le pazienti hanno dimostrato una

E. Arvat

emanuela.arvat@unito.it

1 Divisione di Endocrinologia Oncologica, Dipartimento di Scienze Mediche, Università di Torino, Torino, Italia ridotta capacità massimale aerobica e una ridotta resistenza all'esercizio rispetto alle donne del gruppo di controllo, non modificate dall'assunzione di idrocortisone pre-esercizio. È rilevante notare che tali e non modificabili differenze sono state riscontrate a fronte di livelli circolanti di cortisolo durante la sessione di attività fisica, nelle pazienti dopo la dose aggiuntiva, significativamente più elevati rispetto alle donne del gruppo di controllo. Tale dato suggerisce che l'aumento acuto delle concentrazioni circolanti dell'ormone non è in grado di normalizzare la ridotta risposta delle pazienti iposurrenaliche ad esercizio fisico di breve durata. Lo studio ha inoltre dimostrato una ridotta liberazione di adrenalina e ridotti livelli di glucosio in risposta allo stress fisico nelle pazienti rispetto ai controlli, anch'essi non modificati dall'assunzione di idrocortisone, a suggerire che l'incremento dei livelli di adrenalina e del glucosio siano fattori più importanti rispetto all'incremento del cortisolo nella risposta a uno stress fisico, perlomeno di breve durata.

Lo studio, pur nell'esiguità del campione valutato e nella focalizzazione della risposta a uno stress fisico solo di breve durata, ha dimostrato che in donne con iposurrenalismo primitivo cronico la somministrazione pre-esercizio di una dose aggiuntiva di idrocortisone non si associa ad alcun beneficio in termini di miglioramento dei parametri cardiorespiratori ed endocrino-metabolici, che risultano alterati rispetto ai soggetti sani. Valutazioni su vasta scala e su tipologie differenti di stress fisico sono necessari per chiarire il fabbisogno di glucocorticoide in tali pazienti in relazione all'attività sportiva. 